\title{
Optimal Dual Adaptive Agile Mobile Wireless Power Control ${ }^{\star}$
}

\author{
Minh Hong Ha ${ }^{\mathrm{a}}$, Robert R. Bitmead ${ }^{\mathrm{a}}$, \\ ${ }^{a}$ Department of Mechanical \& Aerospace Engineering, University of California, San Diego, 9500 Gilman Drive, La Jolla CA, \\ 92093-0411, USA
}

\begin{abstract}
Mobile wireless channels change persistently and rapidly, so power control needs to adapt similarly in order to save battery power and mitigate interference. Training signals in every data packet are used to achieve this in current systems, albeit in a suboptimal fashion from a power consumption perspective. This problem is used as the basis for posing and solving in detail an optimal dual adaptive control problem and then deriving heuristic controllers from this optimal solution. Dual refers to the joint requirements of the control signal to probe the system for parameter estimation and to regulate the total energy use; these are conflicting requirements which reveal the complexity of optimal stochastic control in general. The information state is defined, computed and explicitly incorporated into the optimization. Performance and computational load comparisons are made between: the optimal control, the certainty equivalence control, and a simplified heuristic approach. The contribution of the paper is the explicit solution of a classically hard optimal stochastic control problem to expose the role of the information state.
\end{abstract}

Key words: Adaptive control, optimal control, dynamic programming, stochastic control, information state, certainty equivalence

\section{Introduction}

Mobility in wireless communications causes rapid channel variation which, in turn, forces the persistent adaptation of transmission power levels on a per-packet basis. Without this adaptation, battery life is seriously compromised and interference occurs between users. In the PCS1900 standard, rapid (re)acquisition of the correct power (and equalizer) is aided by a training signal present as a mid-amble in the center of every packet sent from the mobile station (MS) to the base station (BS) or vice versa. OFDM based systems use pilot signals in a similar fashion. From a control systems perspective, this is an example of an adaptive control system functioning without human intervention many billion of times per hour. It is highly non-stationary and this paper seeks to explore aspects of optimal adaptive control evident from this context.

In existing applied control laws the BS estimates the received signal-to-noise ratio (SNR) and sends a one-bit power control signal to the MS to increase or decrease current power by $2 \mathrm{~dB}$. We consider the situation in which the MS has knowledge of the SNR estimate and seeks to adjust its trans-

\footnotetext{
‡ This material is based upon work supported by the US National Science Foundation under Grant No. 1102384. Corresponding author Robert R. Bitmead.

Email addresses: hahongminh@gmail.com (Minh Hong Ha), rbitmeadeucsd. edu (Robert R. Bitmead).
}

mission power of the training signal in an optimal adaptive fashion. This brings in duality as introduced by Fel'dbaum [6-8], since higher power facilitates accurate SNR estimation while compromising energy usage. The contributions of the paper are the study of duality in a functioning adaptive control system and the exploration of alternative approaches associated with optimality.

An Optimal Dual Adaptive Control (ODAC) is derived using the information state (Bayesian filter) recursion coupled with the Stochastic Dynamic Programming Equation (SDPE) as in [12]. We believe that this is the first formulation of a meaningful practical persistent adaptation problem in such full detail. The ODAC learning is active and the fade parameter uncertainty is managed inherently in the control. We contrast this with suboptimal controllers by comparing: ODAC; Certainty Equivalence (CE) control; probingenhanced ODAC; and a close-to-optimal heuristic. The comparison is in terms of performance versus computational complexity, since ODAC is effectively intractable ... but optimal.

Most other works on dualized adaptive control (DAC) are suboptimal and borrow from the inclusion of probing into the control signal without optimality. For a thorough survey of suboptimal dual adaptive control methods, the reader is referred to $[9,17]$. There are a number of successful industrial applications of dualized adaptive controllers, [1,18,10,3]. From the perspective of this paper, current cellular mobile 
wireless also falls into the category of effective but suboptimal dualized adaptive power control. Our aim in this paper is to explore optimal dual adaptive control in this context.

\section{Problem formulation}

We treat the problem of MS optimal adaptive transmission power control over a memoryless (i.e. flat) fading additive Gaussian white noise (AGWN) channel with perfect feedback from the BS. We presume the fade is fixed over the packet time but changes packet to packet. The dynamics of this system are given by

$$
\begin{aligned}
& x_{k}=f u_{k-1} \\
& y_{k}=x_{k}+w_{k} .
\end{aligned} \quad x_{0}=f,
$$

for $k=1,2, \ldots, N$, where:

$f$ is the unknown channel fade, which we presume to be constant over one packet transmission time.

$u_{k-1}$ is the transmitted signal chosen by the MS for the current $(k$ th of $N)$ packet training symbol. Given by $u_{k-1}=p_{k-1} a_{k-1}$, where

$a_{k-1}$ is a binary (BPSK) training sequence, $a_{k} \in$ $\{-1,1\}$, known to both MS and BS.

$p_{k-1}$ is the square root of the power of the training signal, known solely to the MS.

$y_{k}$ is the received signal at the $\mathrm{BS}$, and

$w_{k}$ is additive gaussian white noise of known variance $\sigma_{w}^{2}$.

We impose the following assumptions.

\section{Assumption 1}

(1.A) The channel fade can take one of I distinct values, $\{f[1], f[2], \ldots, f[I]\}$.

(1.B) The target signal-to-noise ratio (SNR) at the BS for message transmission is $\gamma^{\star}=6.79 \mathrm{~dB}$, which results in a usable bit-error rate (BER) of $10^{-3}$ for BPSK [13].

(1.C) There are I distinct transmission signal powers corresponding to each possible fade value and $\gamma^{\star}$.

$$
u^{\star}[i]^{2}=p^{\star}[i]^{2}=\frac{\sigma_{w}^{2} \gamma^{\star}}{f[i]^{2}} .
$$

We seek to minimize the training and message power by posing the following problem.

\section{Stochastic Optimal Control Problem}

Minimize the performance index

$$
J=E\left[\sum_{k=0}^{N-1} c_{k}\left(x_{k}, u_{k}\right)+c_{N}\left(\pi_{N}\right)\right]
$$

over all admissible causal feedback control policies,

$$
u_{k}=g_{k}\left(Z^{k}\right) \in\left\{u^{\star}[1], \ldots, u^{\star}[I]\right\} \text {, }
$$

with:

- initial probability mass function (pmf) $\pi_{0}$ of the fade, $f$ - history and posterior pmf of the fade at symbol time $N, \pi_{N}$,

$$
Z^{k}=\left(u_{0}, y_{1}, u_{1}, y_{2}, \ldots, u_{k-1}, y_{k}\right)
$$

- stage cost for $k=0, \ldots, N-1$

$$
c_{k}\left(x_{k}, u_{k}\right)=\left(u_{k}^{\star 2}-\frac{\sigma_{w}^{2} \gamma^{\star}}{f^{2}}\right)^{2},
$$

- terminal cost with $i_{N}^{\star}=\operatorname{argmax}_{i} \pi_{N}$,

$$
c_{N}\left(\pi_{N}\right)=\left(u^{\star}\left[i_{N}^{\star}\right]^{2}-\frac{\sigma_{w}^{2} \gamma^{\star}}{f\left[i_{N}^{\star}\right]^{2}}\right)^{2},
$$

The expectation in (3) is over the $\left\{w_{k}\right\}$ sequence and $\pi_{0}$.

This is a stochastic optimal control problem for choice of training signal powers. Since it involves an unknown parameter, $f$, it might also be labeled adaptive. The solution (to be presented) of this problem involves the information state and Stochastic Dynamic Programming (SDP) [12,2]. It achieves the optimal balance between probing and regulation to minimize $J$ in (3). It also is extraordinarily computationally demanding. Fel'dbaum posed a problem isomorphic to this but was unable to compute the solution with the tools available in the 1960s. This formulation has perfect feedback from BS to MS, which jointly comprise the controller. In the full problem discussed in Section 6 one needs to: separate BS and MS, posit another noisy fading channel connected them, and have each solve a joint optimization problem. This is a degree of difficulty beyond the statement above.

Definition 1 The information state is the conditional probability vector of the state $x_{k}$ at the MS given the available history $\pi_{k}\left(Z^{k}\right)$.

Lemma 1 For the AGWN channel with constant fade described by (1), the information state updates according to

$$
\begin{aligned}
\pi_{k+1}\left(Z^{k+1}\right) & :=T_{k}\left(\pi_{k}\left(Z^{k}\right), y_{k+1}, u_{k}\right), \\
& =\frac{1}{\pi_{k}\left(Z^{k}\right) D\left(y_{k+1}\right) \underline{1}} \pi_{k}\left(Z^{k}\right) D\left(y_{k+1}\right),
\end{aligned}
$$

with: $\underline{1}=\left[\begin{array}{llll}1 & 1 & \ldots & 1\end{array}\right]^{T}$, and diagonal matrix,

$$
D\left(y_{k+1}\right)[i]=\frac{1}{\sqrt{2 \pi} \sigma_{w}} \exp \left[-\frac{\left(y_{k+1}-f[i] u_{k}\right)^{2}}{\sigma_{w}^{2}}\right] .
$$


This is Bayes' Rule

$$
\begin{aligned}
\operatorname{Pr}\left(f=f[i] \mid Z^{k+1}\right) & =\frac{\operatorname{Pr}\left(f=f[i], y_{k+1} \mid Z^{k}\right)}{\operatorname{Pr}\left(y_{k+1} \mid Z^{k}\right)}, \\
= & \frac{\operatorname{Pr}\left(y_{k+1} \mid Z^{k}, f=f[i]\right) \operatorname{Pr}\left(f=f[i] \mid Z^{k}\right)}{\operatorname{Pr}\left(y_{k+1} \mid Z^{k}\right)} .
\end{aligned}
$$

The transformation $T_{k}$ in (7) will appear in the solution via stochastic dynamic programming.

\subsection{Duality}

Figure 1 shows the quadratic stage cost function (5) versus control value $u[i]$, where these values are separated by $2 \mathrm{dBm}$, as is used in practical mobile wireless systems such as PCS-1900. The graph is centered on the correct value $u^{\star}[i]$.

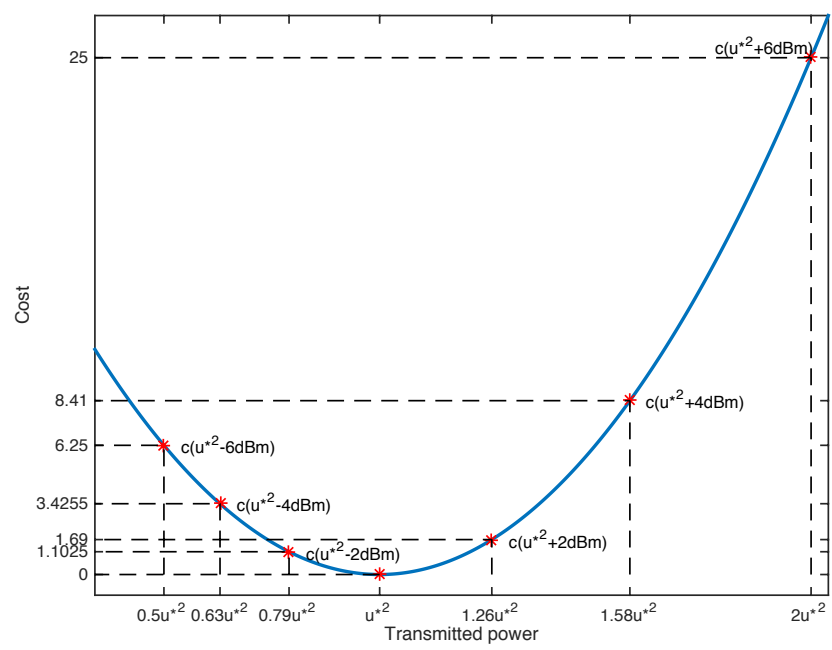

Fig. 1. Quadratic cost (5) for a fixed value of $u^{*}=\frac{\sigma_{w}^{2} \gamma^{*}}{f^{2}}$ and different values of power separated by $2 \mathrm{dBm}$ increments.

Two features are immediately apparent.

(i) The power penalty for incorrect selection of power grows quadratically with distance from $u^{\star}$.

(ii) In the practical $\mathrm{dBm}$ scale, the penalty for choosing too high a power is significantly greater than that for choosing $\mathrm{dBm}$-equivalent too low a power.

Thus there is a penalty for incorrect power choice and this penalty is diminished for cautious lower power selections.

By contrast, it is directly proven from (9) that

$$
\mathrm{E}\left[D\left(y_{k+1}\right)[i]\right]=\frac{1}{2 \sqrt{\pi} \sigma_{w}} \exp \left(-\frac{1}{4 \sigma_{w}^{2}}(f-f[i])^{2} u_{k}^{2}\right) .
$$

The expectation is over $w_{k+1}$. This shows that large values of $u_{k}^{2}$ yield rapid refinement of $\pi_{k+1}$.

\section{Solution via Stochastic Dynamic Programming}

Kumar and Varaiya [12] establish that the optimal control minimizing the performance index (3) is a separated policy - the optimal admissible control depends on the data $Z^{k}$ only through the information state, $u_{k}=g_{k}\left(\pi_{k}\left(. \mid Z^{k}\right)\right)$. The information state is first computed and then the controls chosen. The tasks of estimation and control are then separated.

Let $\mathbf{U}=\left\{u^{\star}[1], \ldots, u^{\star}[I]\right\}$ be the set of possible controls. Then the solution to the stochastic optimal control problem is given by Stochastic Dynamic Programming via Bellman's Equation with $u_{k}^{\mathrm{opt}}$ as the minimizer of the right hand side.

$$
\begin{aligned}
V_{k}\left(\pi_{k}\right)=\min _{u \in \mathbf{U}} E_{k}\left\{c_{k}\left(x_{k}, u\right)\right. \\
\left.+V_{k+1}\left(T_{k}\left(\pi_{k}, y_{k+1}, u\right)\right) \mid \pi_{k}\right\},
\end{aligned}
$$

with terminal value

$$
V_{N}\left(\pi_{N}\right):=c_{N}\left(\pi_{N}\right)
$$

Here $E_{k}$ signifies expectation over $\pi_{k}$ and future information from time $k+1$ onwards. $T_{k}(\pi, y, u)$ is defined by (7).

The core difficulty in solving (11) for $V_{k}$ and $u_{k}^{\text {opt }}$ lies in the application of the information state update $T_{k}(\pi, y, u)$ prospectively forwards across the horizon from time $k$ to $N$. Within the expectation $E_{k}\{\cdot\}$, one must account for the effect of choice of $u_{k+j}$ for $j=0, \ldots, N-k$ on the future values of $\pi_{k+j}$. Not only is Bellman's curse of dimensionality evident in the explosion in $N$ of the number of feasible controls to be considered, but also, for each possible control, the corresponding information state updates and expectation values must be computed. It is the inclusion of these many integrals over the future gaussian densities which dominates the computational burden.

\subsection{Implementation of the Algorithm}

For the specific mobile power problem from Section 2 with a finite set of possible fades and corresponding powers, the terminal value function is given by (12) and the precedent value functions (11) can be expressed as

$$
\begin{aligned}
& V_{k}\left(\pi_{k}\right):=\min _{u_{k} \in \mathbf{U}}\left\{\sum_{i} c_{k}\left(i, u_{k}\right) \pi_{k}[i]\right. \\
& \left.+\int_{y_{k+1}} V_{k+1}\left(T_{k}\left(\pi_{k}, y_{k+1}, u_{k}\right)\right) p\left(y_{k+1} \mid \pi_{k}, u_{k}\right) d y_{k+1}\right\} .
\end{aligned}
$$

The pdf $p\left(y_{k+1} \mid \pi_{k}, u_{k}\right)$ is a mixture of normal densities,

$$
p\left(y_{k+1} \mid \pi_{k}, u_{k}\right)=\sum_{i} p\left(y_{k+1} \mid f=i\right) \pi_{k}[i] .
$$


To obtain the expressions for $V_{N-1}\left(\pi_{N-1}\right)$ and $u_{N-1}$, we first write the terminal value function $V_{N}$ as a function of $\pi_{N-1}$

$$
V_{N}\left(T_{N-1}\left(\pi_{N-1}, y_{N}, u_{N-1}\right)\right)=c_{N}\left(\frac{\pi_{N-1} D\left(y_{N}\right)}{\pi_{N-1} D\left(y_{N}\right) \underline{1}}\right) .
$$

Then

$$
\begin{aligned}
V_{N-1}\left(\pi_{N-1}\right) & =\min _{u_{N-1} \in \mathbf{U}}\left\{\sum_{i} c_{N-1}\left(i, u_{N-1}\right) \pi_{N-1}[i]\right. \\
& \left.+\int_{y_{N}} c_{N}\left(\frac{\pi_{N-1} D\left(y_{N}\right)}{\pi_{N-1} D\left(y_{N}\right) \underline{1}}\right) d y_{N}\right\}
\end{aligned}
$$

Remark: The density (14) is identical to the normalization factor that appears in the information state update (8) so the integral in (11) can be reduced to a simpler form while (12) remains unaltered.

For preceding, $k<N$, value functions, the cancelation of the normalization $\pi_{N-1} D\left(y_{N}\right) \underline{1}$ and the density mixture $p\left(y_{N} \mid \pi_{N-1}, u_{N-1}\right)$ occurs when combined in (13). Note that $V_{N}$ in (12) is not an expectation, unlike the preceding terms. This cancellation of the normalization with the corresponding factor in the density mixture holds true for each precedent term because each is independent of the control over which the minimization is performed and we arrive at the following expanded form of (11). [We use the index $i$ of the state here in place of the state itself.]

$$
\begin{aligned}
V_{N-k}\left(\pi_{N-k}\right)=\min _{u_{N-k} \in \mathbf{U}}\left\{\sum_{i} c_{N-k}\left(i, u_{N-k}\right) \pi_{N-k}[i]+\int_{y_{N-k+1}} \min _{u_{N-k+1} \in \mathbf{U}}\left\{\sum_{i} c_{N-k+1}\left(i, u_{N-k+1}\right) \pi_{N-k}[i] D\left(y_{N-k+1}\right)[i]\right.\right. \\
+\cdots+\left\{\sum_{i} c_{N-1}\left(i, u_{N-1}\right) \pi_{N-k}[i] D\left(y_{N-k+1}[i]\right) \ldots D\left(y_{N-1}[i]\right)\right. \\
\left.\left.\left.+\int_{y_{N}} c_{N}\left(\frac{\pi_{N-k} D\left(y_{N-k+1}\right) \ldots D\left(y_{N}\right)}{\pi_{N-k} D\left(y_{N-k+1}\right) \ldots D\left(y_{N}\right) \underline{1}}\right) d y_{N}\right\} d y_{N-1}\right\} \ldots d y_{N-k+1}\right\} .
\end{aligned}
$$

Remark 1 The computation of the initial control value, $u_{0}^{\star}$, is significantly more costly in calculation time than is the computation of any subsequent control value. Indeed, the computation time is dominated by the initial control value.

Remark 2 The initial control signal is a deterministic function of the initial information state. That is, $u_{0}^{\star}\left(\pi_{0}\right)$. Indeed, each value function $V_{k}$ and optimal control $u_{k}$ is a deterministic function of $\pi_{k}$. This feature can be used to replace the on-line computational intractability by significant offline computation and approximation via look-up tables for $V_{k}$ and $u_{k}$ indexed by $\pi_{k}$, an element of a 3-dimensional simplex in this example. This and other approaches to replacement of the curse of dimensionality by the curse of complexity are discussed in [15].

\section{Heuristics and alternative control laws}

We draw on Remarks 1, 2 to support the following modifications to reduce the computational burden of optimal control.

Heuristic 1 Compute the initial optimal control value $u_{0}^{\star}\left(\pi_{0}\right)$ off-line as a precomputed function of the initial information state vector $\pi_{0}$. Then use a look-up table for this value followed by a reduced-horizon optimal control calculation or other control law.

Concept 2 Propagate the information state from packet end,
$\pi_{N}^{t}$, to next packet initiation, $\pi_{0}^{t+1}$. This can be accomplished by a Markov probability transition matrix, $P_{j k}^{\pi}=$ $\operatorname{Pr}\left(f_{t+1}=f[k] \mid f_{t}=f[j]\right)$, where $P_{j k}^{\pi}$ reflects knowledge of the channel, e.g. Rayleigh fading channel.

$$
\pi_{0}^{t+1}=\pi_{N}^{t} P_{j k}^{\pi}
$$

While we do not simulate Concept 2, we note two properties which justify attention to its information state propagation between packets. Clearly, the refinement of the information states presents a challenge to the control performance because of duality of the optimal control. So providing a concentrated information state is beneficial. Secondly, it is apparent from (8) that a zero element anywhere in $\pi_{k}$ cannot be removed at subsequent times; likewise for a one element. Accordingly, it is important to ensure that any information state degeneracy is removed via (16) before the next packet.

\subsection{Alternative Control Laws}

Beside our main focus on the ODAC solution, we also consider the following alternative suboptimal control laws.

CE: based on the Certainty Equivalence principle. Here the information state $\pi_{k}$ is updated as usual using (8) based on the received signal $y_{k}$, but $u_{k}$ is chosen to be the 
maximal a posteriori probability control.

$$
u_{k}^{\mathrm{CE}}=u^{\star}\left(\underset{i}{\operatorname{argmax}}\left\{\pi_{k}[i]\right\}\right) .
$$

This is a low computational complexity, familiar, and suboptimal controller.

H: based on Heuristic 1 precompute optimal $u_{0}^{\star}\left(\pi_{0}\right)$ then use Certainty Equivalence control through the remainder of the horizon. Since the computation of $u_{0}^{\star}$ takes place off-line and CE control is low complexity, this controller too is low complexity. But it differs from $\mathrm{CE}$ in possessing an optimal initial step.

ODAC-100: based on optimal dual adaptive control with a variation of the cost function achieved by multiplying the terminal cost, $c_{N}\left(\pi_{N}\right)$, by 100. This alters the original cost function by emphasizing the refinement of the information state by the terminal time. Since, in our scenario, we have a training sequence with optimally chosen powers but known to both MS and BS, the expectation is that non-adaptive power control or non-optimal CE adaptive power control will proceed after the training signal. This new cost function attempts to capture the penalty for poor fade estimates at time $N$. ODAC-100 will not in general be optimal for the original ODAC problem. But it should exhibit greater probing.

\section{Computational results}

Using mat $l \mathrm{ab}$ we consider an optimal control problem with the following parameters

Horizon $N=4$.

Fade $f$ takes values in the set $\{-17,-7,-3,0\} \mathrm{dB}$.

Noise sequence $\left\{w_{k}: k=0, \ldots, 4\right\}$ is taken over 10 independent realizations and is the same for each control law. Performance plots are averaged over the 10 realizations.

Noise power $\sigma_{w}^{2}=-5 \mathrm{dBm}$.

Power for signal transmission takes values from a set with each power associated with the corresponding fade value as in (2) with $\gamma^{\star}=6.79 \mathrm{~dB}$.

$\left\{u^{\star^{2}}[1], u^{\star^{2}}[2], u^{\star^{2}}[3], u^{\star^{2}}[4]\right\}=\{18.5,8.9,4.5,1.5\} \mathrm{dBm}$.

Information State $\pi_{0}$ is an under-informed 4-vector

$$
\pi_{0}=[0.35,0.15,0.1,0.4] .
$$

Integration is conducted in (15) using a 20-point trapezoidal rule spanning \pm five standard deviations of the Gaussian density.

Control laws ODAC, CE, H, and ODAC-100 are computed and compared using the ODAC empirical cost function.

$$
J=c_{4}\left(\pi_{4}\right)+\sum_{k=0}^{3} \sum_{i=1}^{4} c_{k}\left(i, u_{k}[i]\right) \pi_{k}[i],
$$

with $c_{k}$ from (5) and $c_{4}$ from (6).

\subsection{Performance and computation time}

The empirical performance index (19) associated with the $a$ priori information (18) and each control policy is tabulated in Table 1 for each value of the true fade $f$. Table 2 indicates the time taken for a simulation with one realization of the noise to complete for each control scheme and with differing time horizons.

\begin{tabular}{|c|c|c|c|c|}
\hline Actual fade: & $f[1]$ & $f[2]$ & $f[3]$ & $f[4]$ \\
\hline ODAC & 21 & 25 & 15 & 14 \\
\hline ODAC-100 & 25 & 35 & 19 & 14 \\
\hline CE & 36 & 34 & 35 & 32 \\
\hline H & 21 & 26 & 15 & 14 \\
\hline
\end{tabular}

Table 1

Average empirical performance $J\left[\times 10^{4}(\mathrm{~mW})^{2}\right]$ from (19) of the control policies and differing real fade values, $f[i]$.

\begin{tabular}{|c|c|c|c|}
\hline Time horizon & ODAC \& ODAC-100 & $\mathrm{CE}$ & $\mathrm{H}$ \\
\hline 4-step & $3300 \mathrm{~s}$ & $7 \mathrm{~ms}$ & $7 \mathrm{~ms}$ \\
\hline 3-step & $42 \mathrm{~s}$ & $0.3 \mathrm{~ms}$ & $0.3 \mathrm{~ms}$ \\
\hline 2-step & $1 \mathrm{~s}$ & $0.2 \mathrm{~ms}$ & $0.2 \mathrm{~ms}$ \\
\hline
\end{tabular}

Table 2

Computation time of the control laws for a single realization of the measurement noise and differing time horizons.

Observations can be made based on Tables 1 and 2 .

- ODAC outperforms the other control laws, although it does so at very considerable computational and complexity cost. The optimality of ODAC is in terms of expectation and definitely need not be evident with every noise sequence. Indeed, within the ten realizations there are some where CE outperforms ODAC for that specific noise realization.

- ODAC-100, even though a result of the full stochastic dynamic programming algorithm, is suboptimal for the ODAC cost. The balance between probing and regulation leans towards probing when compared to ODAC.

- CE displays its suboptimality, reflecting its lack of probing or, equivalently, undue focus on regulation.

- Heuristic policy $\mathrm{H}$ achieves performance between ODAC and CE, but very close to ODAC. Its computational complexity is very close to that of CE.

- Computational time for SDP increases extremely rapidly with increasing SDP time horizon.

- The computational times of ODAC and $\mathrm{H}$ differ by a factor of nearly 500, 000 in the case of 4-step SDP. This demonstrates the benefit of choosing $u_{0}^{*}$ based on $\pi_{0}$. 


\subsection{Examination of control laws}

Figure 2 is a collection of 80 histograms of the optimal control sequences, $u_{0}^{\star}$ through $u_{4}^{\star}$, averaged over the 10 different channel noise realizations. The histograms are indexed by: time 0-4 (also color); control law H, CE, ODAC-100, ODAC; and control signal value $u[j]$ applied. Fade $f[3]=-3 \mathrm{~dB}$ is the actual fade of the channel and $\pi_{0}$ is given in (18).

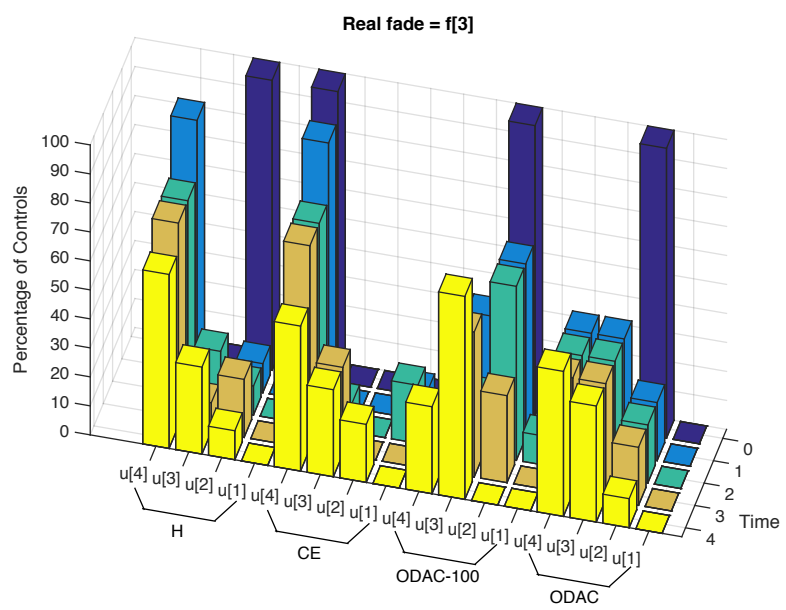

Fig. 2. Histograms of control values averaged over 10 realizations of the noise for each adaptive control scheme vs. time. The real fade is equal to $f[3]=-3 \mathrm{~dB}$.

- The histograms in Figure 2 for time 0 demonstrate Remark 2, that the initial control $u_{0}^{\star}$ for ODAC is a function solely of $\pi_{0}$ and does not depend on the noise realization. This holds for the other three control policies, although each controller selects its own value of $u_{0}^{\star}$. This is shown by a single value of the initial control for each policy occurring for every realization.

- As expected, ODAC-100 is the most reliable in terms of selecting the correct power level by the terminal time, followed by ODAC. This reflects the choice of cost function to emphasize probing.

- This example has a particularly poorly informed $\pi_{0}$ in (18). The actual fade value has a priori probability 0.1 . Indeed, $\pi_{0}$ is rather uninformative and we see that ODAC, (and therefore) $\mathrm{H}$ and ODAC-100 select larger control values early than does $\mathrm{CE}$. This is probing to resolve the actual fade.

The two collections of histograms in Figure 3 illustrate, for each control policy, the evolution of the information state averaged over 10 different noise sequences, with differing real fade values: $f[3]$ (upper) and $f[4]$ (lower). Note that $f[3]$ has low a priori probability in (18), while $f$ [4] has maximal a priori probability. This highlights the control value of the quality of the initial information state. From a mobile communications perspective, where fade estimation is part of every transmitted packet, the propagation of $\pi_{N}$ from one packet to provide as accurate as possible a value of $\pi_{0}$ for the next is important. This demonstrates the substance of Concept 2.
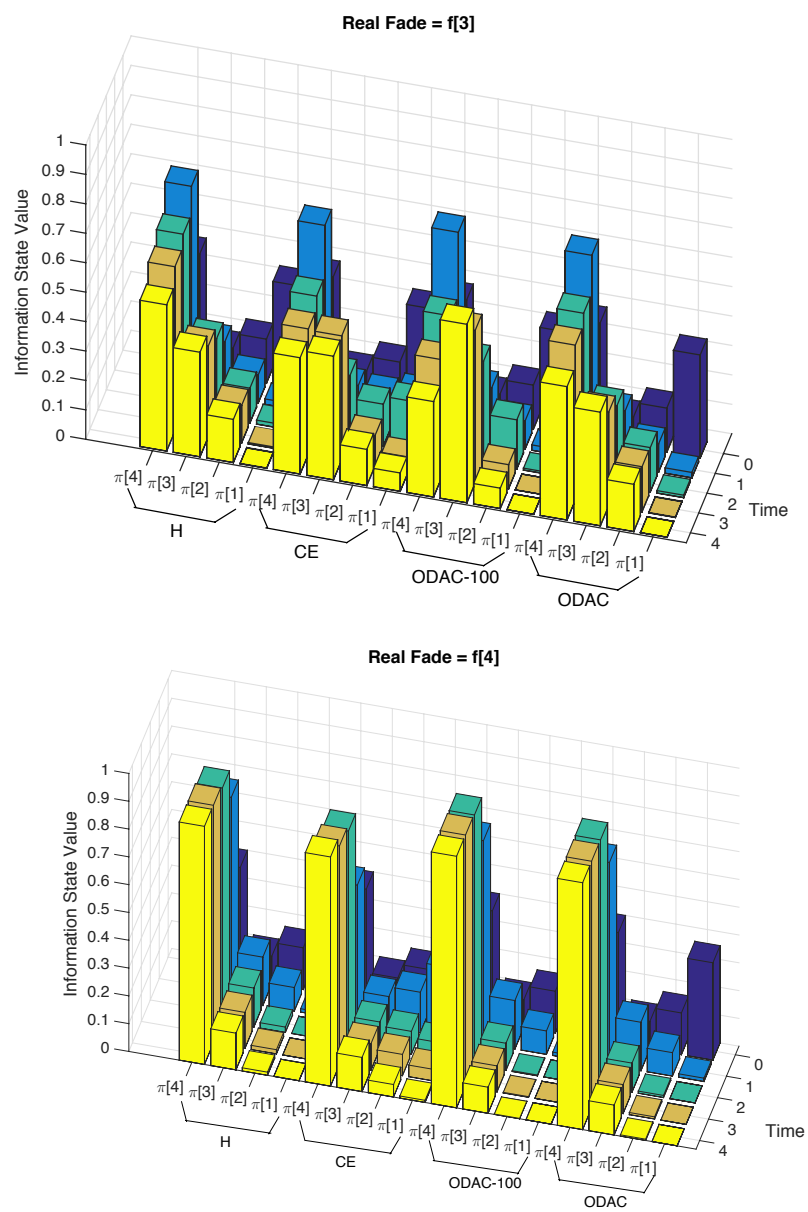

Fig. 3. Evolution of the information state averaged over 10 realizations of the noise for each adaptive control scheme vs. sample time. Upper plot: real fade is $f$ [3]. Lower plot: real fade is $f[4]$.

\section{More complete problem}

A more complete analysis of the mobile wireless power control problem requires extension of the set up developed in Section 2. In practice, the communication link between the MS and BS is not perfect - the signal from MS to BS is: limited in bitrate, corrupted by different channel noise, and is subject to either the same or a different fade. The MS and BS can now be seen as two distinct parts of the controller that communicate. This is a much harder (and therefore even more intractable to optimize) problem since the signaling between MS and BS needs to be incorporated into the ODAC scenario.

In most practical mobile systems, the BS transmits a single bit to the MS, which then interprets this as go up in power by $2 \mathrm{dBm}$ or go down in power by $2 \mathrm{dBm}$ depending on sign. 
Alternative methods have been proposed by others. For example, the MS transmitted power is changed by a multiple of $2 \mathrm{dBm}$ steps based on the number of consecutive requests from BS to change the transmitted power in one direction [4]. In [14], the MS changes the transmitted power at a step size formulated as the product of a basic step size, based on its velocity, and a weighing factor chosen by an unexplained rule based on the 3 most recent command bits sent from the BS. In [11], the authors use a fixed step size based on the difference between the actual link SNR and the target SNR collected over a certain period of time. The common feature of these approaches is that there is no theoretical basis for choosing the signaling schemes.

The central difficulty of the full problem, in which the signaling solution is determined as part of the optimization, is that both the BS and MS need to maintain an information state describing their own uncertainties as well as an information state describing the other's information state and so on ad infinitum. This beliefs propagation complicates the problem and renders it intractable in the framework of SDP. A suboptimal approach with manageable information state surrogates may be made using the notion of bounded rationality [16] in stochastic cooperative game theory.

Dudebout and Shamma [5] consider a multi-agent problem, where the agents neglect the fact that they are mutually dependent through the environment and adapt their decisions on the basis of empirical observations from the environment. This process allows each agent to pose a Markov decision problem instead of a partially observed Markov decision problem. Given sufficient measurements, each agent develops its own Markov model based on empirical observations that changes the environment. Due to the changes, the agents then compute new models and adjust their actions. The authors call this empirical evidence equilibrium. In our context of mobile communications, such approaches are both suboptimal and profligate in their use of data. But they do indicate the complexity of the solution process. The requirement of repeated rapid adaptation precludes consideration of such slow schemes.

\section{Conclusion}

The power control problem for mobile wireless is an example of a functioning adaptive control system, which operates routinely billions of times per second without human intervention. Primarily it is focused on energy usage (battery charge management) and interference mitigation. In this paper we have posed an optimal dual adaptive version of the problem dealing solely with energy minimization. To our knowledge, this is the first computational example of a truly optimal dual adaptive control solution based on a practical problem. It is of interest because of its horrendous computational complexity and its optimality. However, Heuristic 1 yields close-to-optimal performance with limited (on-line) computational demand. Similarly, Concept 2 suggests an approach to power control which seeks to improve packet-topacket fade estimate retention.

\section{References}

[1] B. Allison, J. Ciarniello, P. Tessier, and G. Dumont. Dual adaptive control of chip refiner motor load. Automatica, 31(8):1169-1184, 1995.

[2] Y. Bar-Shalom. Stochastic dynamic programming: caution and probing. IEEE Trans. Automat. Contr., AC-26(5):1184-1195, Oct. 1981.

[3] M. Bugeja and S. Fabri. Dual adaptive dynamic control of mobile robots using neural networks. IEEE Trans. on Systems, Man, and Cybernetics, Part B, 39(1):129-141, 2009.

[4] M. G. Cea, G. C. Goodwin, and T. Wigren. Model predictive zooming power control in future cellular systems under coarse quantization. In IEEE Veh. Tech. Conf. (VTC Fall), pages 1-5, Quebec City, QC, Sept. 2012.

[5] N. Dudebout and J. S. Shamma. Empirical evidence equilibria in stochastic games. In IEEE 51st Annual Conference on Decision and Control, pages 5780-5785, Maui, Hawaii USA, Dec. 2012.

[6] A. A. Fel'dbaum. Dual-control theory, Part I. Automation \& Remote Control, 21:874-880, 1960

[7] A. A. Fel'dbaum. Dual-control theory, Part II. Automation \& Remote Control, 22:1-12, 109-121, 1961.

[8] A.A. Fel'dbaum. Optimal Control Systems. Academic Press, New York, NY, 1965.

[9] N. Filatov and H. Unbehauen. Adaptive dual control. Springer, New York, 2004.

[10] A. Ismail and G. Dumont. Dual adaptive control of paper coating. IEEE Trans. on Control Systems Technology, 11(3):289-309, 2003.

[11] M. R. Khan and P. C. Jain. A simple modified fixed step size power control algorithm for CDMA cellular systems. In International Muitimedia, Signal Processing and Communication Technologies, IMPACT 09, pages 134-137, Aligarh, March 2009.

[12] P. R. Kumar and P. Varaiya. Stochastic Systems: Estimation, Identification, and Adaptive Control. Prentice-Hall, Englewood Cliffs, NJ, 1986.

[13] E. A. Lee and D. G. Messerschmitt. Digital Communication. Kluwer Academic Publishers, The Netherlands, 2nd edition, 1990.

[14] H.J. Lee and D.H. Cho. A new adaptive power control scheme based on mobile velocity in wireless mobile communication systems. In 53rd IEEE Vehicluar Technology Conference, volume 4, pages 2878-2882, 2001.

[15] W. M. McEneaney. A curse-of-dimensionality-free numerical method for solution of certain HJB PDEs. SIAM Journal on Control and Optimization, 46(4):1239-1276, 2007.

[16] A. Rubinstein. Modeling bounded rationality. MIT Press, Cambridge, MA, 1998.

[17] B. Wittenmark. Adaptive dual control methods: An overview. Proceedings of the $5^{\text {th }}$ IFAC Symposium on Adaptive Control and Signal Processing Budapest, pages 67-72, 1995.

[18] B. Wittenmark and C. Elevitch. An adaptive control algorithm with dual features. In Proc. 7th IFAC/IFORS Symp. on Identification and System Parameter Estimation, pages 587-592, York, UK, 1985. 